\title{
Simulation modeling for heart attack patient by mapping cholesterol level
}

\author{
Jossy P. George ${ }^{1}$, Suhas M. Gaikwad ${ }^{2}$ \\ ${ }^{1}$ Director and Dean at Christ Deemed to be University, India \\ ${ }^{2}$ Researcher at Christ Deemed to be University, India
}

\begin{tabular}{l} 
Article Info \\
\hline Article history: \\
Received Jul 27, 2019 \\
Revised Sep 28, 2019 \\
Accepted Oct 15, 2019 \\
\hline Keywords: \\
Heart disease \\
Mathematical modeling \\
Simulation \\
System dynamics \\
Vensim
\end{tabular}

\begin{abstract}
Cholesterol is a complex structural material made up of four-fused hydrocarbon rings. There is a hydrocarbon tail linked at one end of the structure, while the hydroxyl group linked to each other on the other end. To one end of the structure, a hydrocarbon tail linked and to the other end, a hydroxyl group linked to each other. High cholesterol level is one among the major risk factors of a heart attack. It is feasible to compute and control the cholesterol level of a cardiovascular patient by making use of intended Mathematical modeling in System Dynamics (S.D.). Moreover, by simulating proposed set of equations for a heart attack patient, recovery accomplished at a faster pace. Because of S.D., a substantial amount of reduction in the patient's Cardiovascular Disease achieved by control over the sterol level of the heart patient. This simulation modeling is an attempt made in translational research domain and is useful in the healthcare industry health care industry. It will minimize the risk of heart stroke and maintain a healthy life.
\end{abstract}

Copyright $(0) 2020$ Institute of Advanced Engineering and Science. All rights reserved.

\section{Corresponding Author:}

Suhas Machhindra Gaikwad, Department R \& D 30 Valor Court, Dasve Cir, Lavasa, Maharashtra 412112, India.

Email: suhasg2009@gmail.com

\section{INTRODUCTION}

Nevertheless, a Heart attack occurs when blood flow decreases or blood stopped from entering the heart, thereby causing damage to the heart muscle. The above-mentioned condition known as heart attack or cardiovascular disease. This disease is currently very common and it might lead to the death of a patient. About 610,000 people die of heart disease in the USA. Thereafter, 1 out of 4 deaths caused due to a heart attack. Thus, Lifestyle factors such as high blood pressure, diabetes, smoking, obesity, lack of physical activity can lead to a heart attack [1]. Now, apart from shortness of breath or fatigue, symptoms for heart attack include pressure, tightness [2] and strain in areas of the upper body like chest and arms. Symptoms, as well as the severity of pain, may vary from person to person [3]. Consequently, some people develop mild pain some while some others will experience severe pain. However, a few may not have any symptoms at all while for some the first symptom may be a cardiac arrest. Moreover, increasing the Heart attack symptoms one has a higher risk of cardiovascular disease. A few cardiac attacks occur immediately while in many cases the victims have symptoms or signs such as indigestion or pain a few hours or days in advance. Also in some cases, a cardiac attack shows indication hours or days before the actual heart attack occurred [4]. The initial symptoms will be immediate chest pain occurring for a fraction of seconds when blood supply to the heart stopped [5]. A cardiac arrest occurs when a couple of coronary arteries blocked due to sterol accommodation in the arteries [6]. A large amount of sterol blockage in the arteries causes an interruption in blood supply to the heart resulting in a condition known as cardiovascular disease, the root cause of heart attack [7]. Thus, congeal of blood occurs during attack [8]. If blood clots and the clot is large enough then surely it will 
try to barricade blood streamflow. If blood if not supposed to enter the heart then the heart is in deficiency condition of oxygenated blood and nutrient supply [9]. The barricade to bloodstream will cause a partial or complete blockage. A large or full block demonstrate ST-elevation myocardial infarction (STEMI) [10]. A small or partly blockage demonstrates a non-ST elevation myocardial infarction (NSTEMI). Another cause may be spasm of coronary arteries that abandon the blood flow to a part of heart muscle [11]. However, no simulation modeling available until for the Heart patient. Thus, the need for advanced simulation modeling in real-time for the Heart patient. Moreover, the above-mentioned problem addressed for heart patient as well as for normal people. Furthermore, heart patient recovery rate estimated in days after consumption of cholesterol added food by heart patient. Thus, the patient's food consumption and its impact on heart cholesterol level measured in real-time. Also, suggested the recovery rate in days for a Heart patient.

a) Cardiovascular Disease Liability Aspect following:

This section discusses the liability aspect of cardiovascular disease [12], which includes the

Adulthood: Men who are around 45 and women who are 55 years and above are more likely to have a heart attack. Adulthood may be one of the reasons behind cardiovascular among senior citizens.

Nicotine: Nicotine consumption is another valid reason. For instance, smoking, whether it is active or passive involves the risk of attack. People in the age group of 25 to 35 are mostly involved in active or passive smoking.

Hypertension: Hypertension occurs due to obesity and it blocks the arteries furthermore causing risk of cardiovascular disease.

A high percentage of sterol: Having a maximum level of low-density lipoproteins (LDL) sterol termed as bad sterol is most feasible to narrow the arteries. The maximum level of triglycerides, a type of blood fat also increases the risk of cardiovascular disease. However, a high-density lipoprotein (HDL), termed as good sterol, can lower the risk of cardiovascular disease.

Overweight: High sterol level is by overweight. It also indicates high triglycerides levels, high blood pressure, and diabetes. Losing just 10 percent of weight can lower the risk of cardiovascular disease.

Type two Diabetes: Insulin obtained by secretion of pancreatic glands. When the body does not respond to insulin properly or there is less insulin production in the body, it causes the blood sugar level to rise and thereby increases the risk of a heart attack.

Holometabolism complex: Being overweight is one reason for high blood pressure and high sugar level. Obesity, in some percentage, is directly proportional to high sugar level and high blood pressure. It then leads to an increase in the risk of cardiovascular disease.

Genealogy of cardiovascular disease: A person whose grandparents or parents have a history is more likely to have a heart attack. Inspecting the family history of a patient concerning cardiovascular disease can be very useful. If a few members of a family are suffering from cardiovascular disease, then the chances of susceptibility to heart diseases are less.

Absence of physical exercise: Lack of physical activity can lead to high blood pressure and obesity. Those who exercise regularly have a good metabolism and are physically fit.

Stress: How to deal with stress is a major reason for an increase in cardiovascular disease. Accent can be useful in the diagnosis of cardiovascular disease.

Antiquity of preeclampsia: Pregnant women can have this type of risk for heart attack, which may lead to the lifetime risk factor of a heart attack. In pregnancy, pregnant women tend to have a major risk of Antiquity of preeclampsia.

b) Complication of Heart Attack.

Damage done to the heart can lead to the following abnormal conditions [13].

Unusual heart rhythm: If an electrical short circuit occurs then it can develop abnormal heart rhythm, some of which can be serious or even fatal.

Heart failure: An attack might damage such a lot of heart tissues that the remaining tissues may not be likely to pump out blood from the heart. Heart failure can be temporary or it can be a chronic condition.

Sudden cardiac arrest: Anthemia can be caused without any warning ie. A heart can stop due to electric short function. Heart attack increases the risk of sudden cardiac arrest in the chest.

\subsection{Contribution}

In this paper, System Dynamics (S D) Simulation model for identifying a patient suffering from Heart Attack proposed. Considering the different stock variable are taken into consideration like Sterol per day $\mathrm{CH}$ (Cholesterol level of a given item) Infectivity LDL HDL TRI Time to recover Total people NonCholesterol About to recover. Although all the stock have different values, that everything will depend on the patient's data. Enhancement in the real-world application system achieved. Vensim simulation used for dynamic simulation modeling and achieved by the feedback control loop. 
a) Top-notch quality with consistency.

b) Instant Connection to input and refined calibration is included.

c) Instant feedback loop output with simulation in SynthSim.

d) Malleable replica model for assembly.

e) Model inquiry consisting of escalation and Monte Carlo replica.

In simulation and modeling technology, the industry-leading software in Vensim. Simulation model requires a different set of proposed equations for the heart patient, concerning per patient's meat consumption rate. Simulation results of patient mapped in terms of days and recovery are noted.

\subsection{Organization}

The introduction of paper given in Section 1. NCRT Model. The actual working of the Vensim Simulation NCRT model shown in Section 3. Section 4 includes the results of NCRT simulation and Section 5 concludes the work.

\subsection{Literature Survey}

S.M. Gaikwad and R.Joshi used AHP, ANP, and Data Mining techniques to predict the food intake for Heart patient and proposed new algorithm for data mining and clustering. Obtained the verification and validation of results of the AHP, ANP with clusters formation. Moreover, they used medical dataset. Dataset of the diabetic patient along with Ben and Jerry ice cream from the Satchrunch website. Initially, the AHP Mathematical decision-making tool is used. AHP gives rise to a suitable decision based on the criteria. Criteria defined for the ice cream and Diabetic patient. AHP will suggest which particular Ben and Jerry ice cream given to a particular diabetic patient. While ANP is similar to AHP. ANP is possible using a feedback loop to AHP. Therefore, the results of the ANP gained by implementing a feedback loop on AHP. ANP uses the multiple criteria level. However, the criteria level in the AHP is limited up to seven. AHP and ANP results obtained. ANP result validates the results of AHP. Same recommendation of ice cream to a diabetic patient obtained from the result AHP. In Data Mining domain, a new algorithm known as Modified Cluster Formation algorithm (M-CFBA) algorithm proposed. M-CFBA algorithm will give result in the cluster formation form. So a cluster of Ben and Jerry ice cream and Diabetic patient obtained. Moreover mapping of a cluster of ice cream with a cluster of diabetic patient done. Cluster mapping will validate the results of the AHP and ANP results. While on Sir Model has given an idea regarding how to use the Susceptible, Infect able and recovery (SIR) Model. The model illustrates how to use Simulation tools for modeling. Modeling consists of mapping proposed set of infected people with a set of non-infected people. Obtain the recovery rate. It is accessible and effortless to use the System Dynamics Model for the infected people and non-infected people along with to achieve the recovery rate of infected and non-infected people. AHP has started the ice cream recommendation to the patient. AHP recommendation results validated by results of ANP. M-CFBA algorithm has given a cluster of ice crema and diabetic patient. Cluster mapping of M-CFBA will give the validation of the results of the AHP and ANP. While Simulation modeling will give the accurate prediction of mapping of Ice cream with a diabetic patient.

\section{ADVANCEMENT OF SIMULATION MODELING FOR HEART PATIENTS}

A new advanced Simulation model known as Non-Cholesterol patients, Cholesterol patients, Recovery rate and Total people (NCRT) Model proposed in this research work. The full form for NCRT model is Non-Cholesterol patients Cholesterol patients Recovery rate Stock variable for total count model. The above-mentioned model can have a set of equations. These equations can fall in a few categories like equations for the non-cholesterol or normal people, followed by the equation for the cardiovascular disease patient, total people.

\subsection{Developing Mathematical Statement for NCRT Model}

The f NCRT model contains a set of the equation in four parts. The first section of equation pertains to patients with cardiovascular disease. Next set of equations are for the normal people or non-infected people, followed by total people and recovery rate equations. Simulated parameters for the normal people and heart patients are considered and data taken from the Stat crunch website.

\subsubsection{Simulation Parameters Non-Cholesterol (Normal people) Patient (N) [14]}

Normal or healthy people can have different simulated parameters concerning cardiovascular disease. Simulated parameters arranged in the form of the equation as stated.

$$
\mathrm{dn} / \mathrm{dt}=\mathrm{\gamma} * \mu *(\mathrm{~N} / \mathrm{T}) *[(\mathrm{LDL}+\mathrm{HDL})+(\mathrm{TRI}) / 5]
$$


Where $\mathrm{V}=$ Sterol for every twenty-four-hour or Cholesterol rate $(200 \mathrm{mg} / \mathrm{dl})$

$\mu=$ Infection parameter concerning time i.e. probability of the influence $(0.5)$

$\mathrm{N}=$ Normal people (990 unit people)

$\mathrm{T}=$ Total count for stock variable (unit people)

$\mathrm{LDL}=$ Sterol consumption for twenty four hour $(220 \mathrm{mg} / \mathrm{dl})$

$\mathrm{HDL}=$ Mark to achieve for Cholesterol level $(140 \mathrm{mg} / \mathrm{dl})$

Tri $=$ Triglycerides $/ 5$ and Total cholesterol=LDL+HDL+ (TRI/5)

Normal people rate " $\mathrm{dn} / \mathrm{dt}$ "concerning time are taken into consideration. Both the sides of the equation balanced. Containing product of the sterol per twenty-four-hour " $\mathrm{V}$ ", probability " $\mu$ ", into a product of Non-Cholesterol patients "N" to the Total people "T" while its product is multiplied with the Total cholesterol=LDL+HDL+ (TRI/5).

\subsubsection{Simulation Parameters for Heart Attack Patient (S) [14]}

Cardiovascular patients have the criterion in regards to cardiovascular disease for proposing an equation. Simulated parameters considered as the stock variable changing with time. Formation of the equation for cardiovascular patient shown as under. Different stock variables for the cardiovascular patient is considered.

$$
d s / d t=\mathrm{V}^{*} \mu *(\mathrm{~N} / \mathrm{T}) *[(\mathrm{LDL}+\mathrm{HDL})+(\mathrm{TRI}) / 5]-\mathrm{CP} * \mathrm{CH}
$$

The same equation used for the cardiovascular disease patient "as like the normal people as described in section 2.1.1. The only difference between the equations is the subtraction of the Sterol intake "CP" into Sterol rate of the patient "CH". Here CP, the Sterol heart forbearing (10 stock variable count), Sterol content from food (normally $130 \mathrm{mg} / \mathrm{dl}$ ) and remaining stock variable are the same as represented by above-mentioned (1).

\subsubsection{Simulation Stock Variable for Rate of Recovery (R) [14]}

Criterion regarding the recovery rate stated as follows considering the different simulation parameters like intake of sterol per day in the food item.

$$
\mathrm{dr} / \mathrm{dt}=\mathrm{cp} * \Theta
$$

Now the recovery rate that is recovery parameter " $r$ " with stock variable concerning time obtained by multiplication of the sterol intake of a particular day "cp" to the Recovery rate " $\Theta$ ". Where = Recovery rate (1/365 unit days). Here also values and meanings for the rest of the terms in the equation are the same as that of (2)

\subsubsection{Stock Variable for Total Count (T) [14]}

Stock variable regarding the total count calculated as shown. It is the total input calculated with the input of criterion like total count, normal people cardiovascular disease and finally recovery rate.

$$
T(t)=N(t)+C(t)+R(t)
$$

Here, $\mathrm{T}(\mathrm{t})=$ Total count of stock variables

$\mathrm{N}(\mathrm{t})=$ Normal people

$\mathrm{C}(\mathrm{t})=$ Cardiovascular disease infected forbearing

$\mathrm{R}(\mathrm{t})=$ Recovery rate of vensim simulation finally, total people "T $(\mathrm{t})$ " equation obtained by summing up the number of Non-cholesterol "N" (t) people; cholesterol patients "C (t)" and recovery of people "R (t)". The value $\mathrm{T}(\mathrm{t})$ involves a simple summation of the non-cholesterol, cholesterol, and total people.

\section{SIMULATION MODELLING OF NCRT MODEL FOR HEART PATIENT}

The simulation model contains four major sections [15]. The first section contains normal people. It is in the feedback loop given to the cardiovascular disease people [16]. While in between loop several stock variable are involved [17]. They are Cholesterol per day, CH, Infectivity, LDL, HDL, TRI, Time to recover, Total people, Cholesterol, Cholesterol, about to recover [18]. In the next section, about to recover is in the loop with the normal people, cardiovascular disease people and all stock variables [19]. The final section contains the total number of people including all the connection from the stock variables. In addition, normal people, heart patient and about to recover. Moreover, recovery rate. 
In Figure 1 Functional NCRT model makes use of the below-mentioned stock variable parameters:

a) Sterol per day

b) $\mathrm{CH}$ (Cholesterol level of a given item)

c) Infectivity

d) LDL

e) HDL

f) TRI

g) Time to recover

h) Total people

i) No Cholesterol

j) Cholesterol

k) About to recover

All the above-mentioned parameters known as stock variables with the changing values. All the stock variables given in the feedback loop. The meanings for the rest of the terms are the System Dynamics Model for the cardiovascular disease patient.

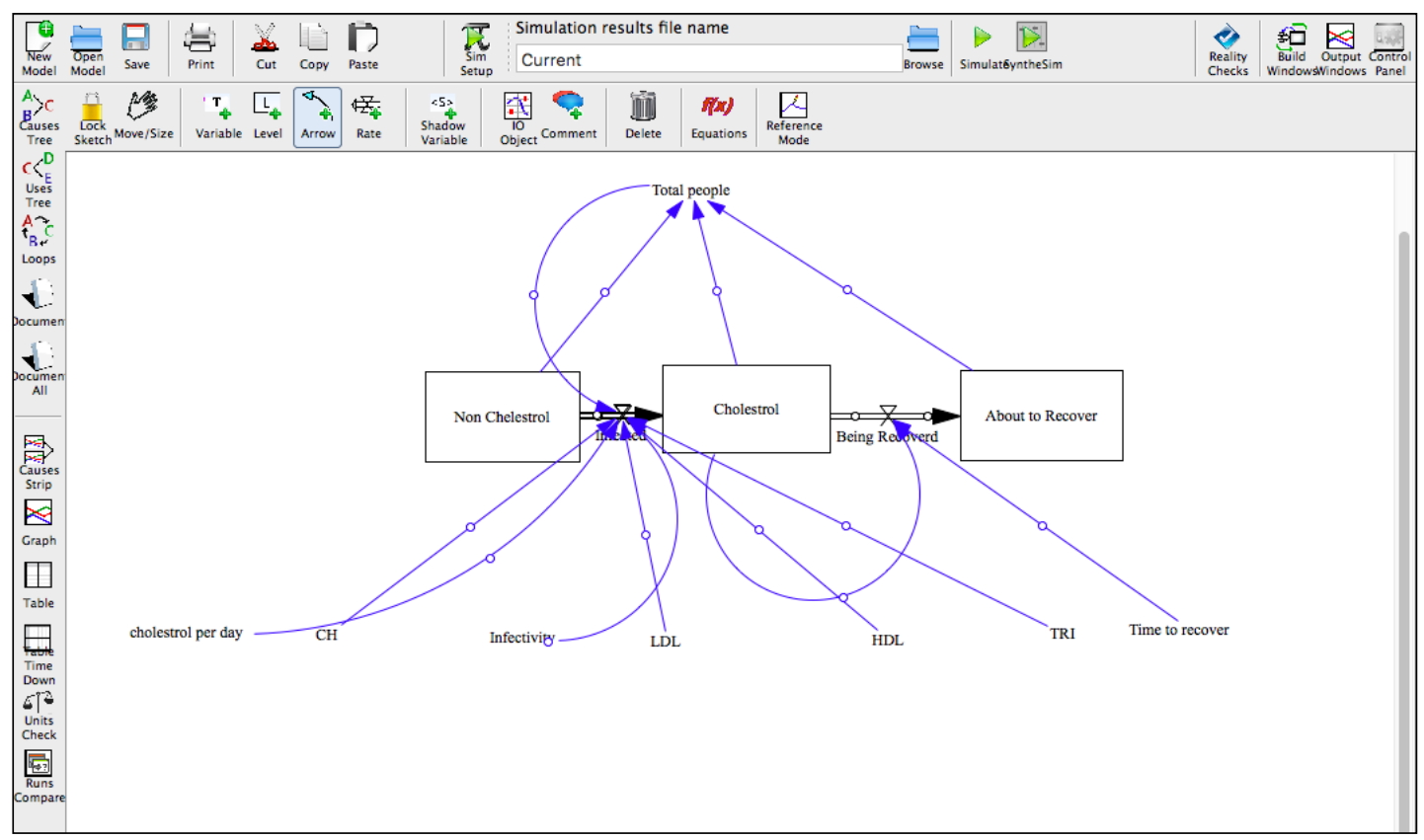

Figure 1. Simulation modelling NCRT model for heart attack patient

\section{RESULTS OF SIMULATION NCRT MODEL FOR HEART PATIENT}

Development obtained from the Vensim simulation model and. Monte Carlo simulation results produced. The graph obtained is several people vs the time of recovery. Plotted graph results show that three different lines are used. The blue color line represents normal people graph vs time to recover. While the green color line represents cardiovascular disease, patient vs time to recover in days. Finally, the red color line shows a constant recovery rate after a few days.

In Figure 2 simulation results obtained from the NCRT model for three different types of graphs. Firstly, the blue color graph line is the simulated result of the non-infected person or normal people [20]. Green color graph line shows the simulation result for the patient having or facing the cardiovascular disease problem. Finally, yet importantly the red color line known as the recovery rate line. Non-infected people that normal people whose recovery rate have shown through the graph and the number of days it will be required to get to a normal level [21]. It means if a patient suffering from cardiovascular disease and consuming food enriched with the sterol then how many days it will take to recover from food intake [22].

The Figure 2 represents the figures all the different colors lines of $\mathrm{CH}$ (cholesterol level) representation. That is the value of sterol in given food content as $73 \mathrm{mg}$ in the regular meat and sterol intake for the day on an average of the patient headcount is $200 \mathrm{mg} / \mathrm{dl}$ and whose age is 71 years [23]. Simulated result of the blue color graph indicates that normal people will take 3 days to recover [24]. Simulated color in 
the green graph shows that the sterol people will take 35 days to recover. While the digit in the number format is as $73 \mathrm{mg} / \mathrm{dl}$ simulated red color graph indicates that recovery achieved after 45 days if everything is steady.

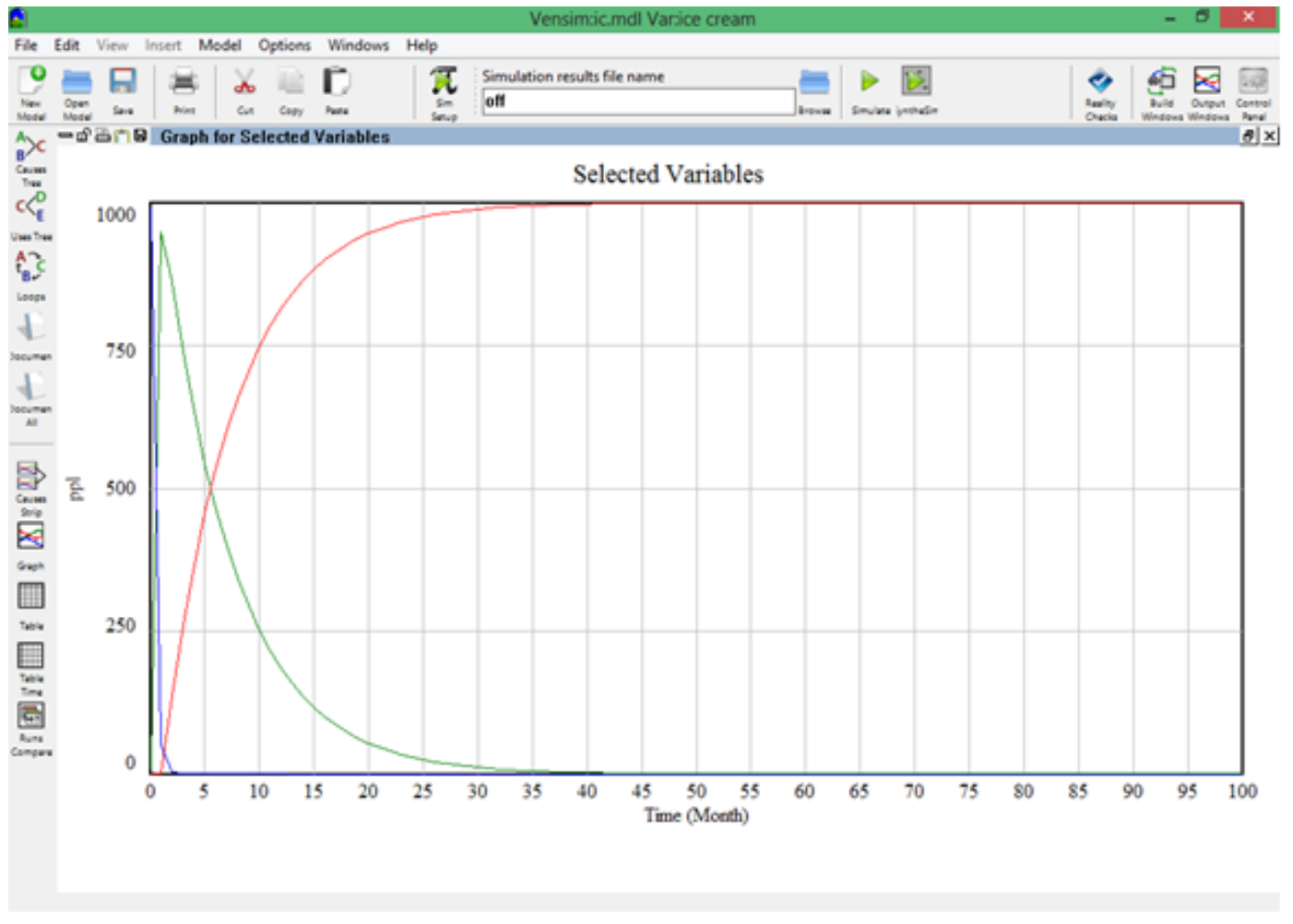

Figure 2. Simulation modelling NCRT model results for heart patient

\section{CONCLUSION}

Thus, till date no simulation modeling available for a Heart patient. For the measurement of the Heart patient cholesterol level based on the food intake. Moreover, the above-mentioned problem addressed for heart patient as well as for normal people. The simulation uses sterol as the main content for modeling and it did on normal people and a heart patient and recovery rate obtained. After simulating the model Vensim Simulation, results obtained. Results show that sterol content in the food of a particular day taken into consideration if the cholesterol level in the food item is varied then the recovery rate for the patient also varies. However, the modeling based on the food item for the cardiovascular disease patient done with advanced NCRT model. Also for diagnosis of heart patients with cholesterol, level in food achieved through simulation. As described in the paper no modeling is available until the date for heart patient based on the food intake so this model will be helpful to give the required results. The suitable food item required for the heart patient simulated with two different conditions of food intake and where are the results. An attempt made based on transitional research on food and useful in health industry domain. Therefore, a heart patient can get surety about food items they can consume, hence maintaining a healthy lifestyle.

\section{REFERENCES}

[1] Vanderby, S., Carter, M., Noseworthy, T. and Marshall, D. (2015). "Modeling the complete continuum of care using system dynamics: the case of osteoarthritis in Alberta". Journal of Simulation, 9(2), pp.156-169.

[2] Marshall, D., Burgos-Liz, L., IJzerman, M., Osgood, N., Padula, W., Higashi, M., Wong, P., Pasupathy, K. and Crown, W. (2015). "Applying Dynamic Simulation Modeling Methods in Health Care Delivery Research The SIMULATE Checklist: Report of the ISPOR Simulation Modeling Emerging Good Practices Task Force". Value in Health, 18(1), pp.5-16.

[3] Valenza, G., Citi, L., Gentili, C., Lanata, A., Scilingo, E. and Barbieri, R. (2015). "Characterization of Depressive States in Bipolar Patients Using Wearable Textile Technology and Instantaneous Heart Rate Variability Assessment". IEEE Journal of Biomedical and Health Informatics, 19(1), pp.263-274. 
[4] Messori, M., Toffanin, C., Del Favero, S., De Nicolao, G., Cobelli, C. and Magni, L. (2016). "Model individualization for the artificial pancreas". Computer Methods and Programs in Biomedicine.

[5] Chen, S., Chen, Y., Xu, L., Matei, N., Tang, J., Feng, H. and Zhang, J. (2015). "Venous system in acute brain injury: Mechanisms of pathophysiological change and function". Experimental Neurology, 272, pp.4-10.

[6] Zulkepli, J. and Eldabi, T. (2015). "Towards a framework for conceptual model hybridization in healthcare". 2015 Winter Simulation Conference (WSC).8] Palma, A., Lounsbury, D., Schlecht, N. and Agalliu, I. (2015). A System Dynamics Model of Serum Prostate-Specific Antigen Screening for Prostate Cancer. American Journal of Epidemiology, 183(3), pp.227-236.

[7] Zulkepli, J. and Eldabi, T. (2015). "Towards a framework for conceptual model hybridization in healthcare". 2015 Winter Simulation Conference (WSC).

[8] Marshall, D., Burgos-Liz, L., IJzerman, M., Crown, W., Padula, W., Wong, P., Pasupathy, K., Higashi, M. and Osgood, N. (2015). "Selecting a Dynamic Simulation Modeling Method for Health Care Delivery Research-Part 2: Report of the ISPOR Dynamic Simulation Modeling Emerging Good Practices Task Force". Value in Health, 18(2), pp.147-160.

[9] Hussin Alsonosi Omar and Y. Abu Hasan, "Numerical simulations of an SIR epidemic model with random initial states," Science Asia, vol. 39S, p. 42, 2013.

[10] Esensoy, A. and Carter, M. (2015). "Health system modelling for policy development and evaluation: Using qualitative methods to capture the whole-system perspective". Operations Research for Health Care, 4, pp.15-26.

[11] Yu, W., Li, M., Ge, Y., Li, L., Zhang, Y., Liu, Y. and Zhang, L. (2015). "Transformation of potential medical demand in China: A system dynamics simulation model". Journal of Biomedical Informatics, 57, pp.399-414.

[12] Lounsbury, D., Schwartz, B., Palma, A. and Blank, A. (2015). "Simulating Patterns of Patient Engagement, Treatment Adherence, and Viral Suppression: A System Dynamics Approach to Evaluating HIV Care Management". AIDS Patient Care and STDs, 29(S1), pp.S55-S63.

[13] Lyons, G. J., \& Duggan, J. (2015). "System dynamics modelling to support policy analysis for sustainable health care". Journal of Simulation, 9(2), 129-139. doi:10.1057/jos.2014.15.

[14] Lyons, G. J., \& Duggan, J. (2015). "System dynamics modelling to support policy analysis for sustainable health care". Journal of Simulation, 9(2), 129-139. doi:10.1057/jos.2014.

[15] Gaikwad, S. M., Joshi, R. R., \& Mulay, P. (2015). "System Dynamics Modeling for Analyzing Recovery Rate of Diabetic Patients by Mapping Sugar Content in Ice Cream and Sugar Intake for the Day". Advances in Intelligent Systems and Computing Proceedings of the Second International Conference on Computer and Communication Technologies, 743-749. doi: 10.1007/978-81-322-2517-1_71.

[16] Gaikwad, S. M., Mulay, P., \& Joshi, R. R. (2015). "Mapping With The Help Of New Proposed Algorithm And Modified Cluster Formation Algorithm To Recommend An Ice Cream To The Diabetic Patient Based On Sugar Contain In It". International Journal of Students Research in Technology \& Management, 3(6), 410. doi:10.18510/ijsrtm.2015.366

[17] Gaikwad, S. M., Mulay, P., \& Joshi, R. R. (2015). “Analytical Hierarchy Process to Recommend an Ice Cream to a Diabetic Patient Based on Sugar Content in it". Procedia Computer Science, 50, 64-72. doi:10.1016/j.procs.2015.04.062

[18] M., S., R., R., \& Mulay, P. (2015). "Cluster Mapping with the help of New Extended MCF Algorithm and MCF Algorithm to Recommend an Ice Cream to the Diabetic Patient". International Journal of Computer Applications, 127(6), 25-27. doi: 10.5120/ijca2015906399

[19] Gaikwad, S. M., Joshi, R. R., \& Kulkarni, A. J. (2016). "Cohort Intelligence and Genetic Algorithm Along with AHP to Recommend an Ice Cream to a Diabetic Patient”. Swarm, Evolutionary, and Memetic Computing Lecture Notes in Computer Science, 40-49. doi: 10.1007/978-3-319-48959-9_4

[20] Gaikwad, S. M., Joshi, R. R., \& Mulay, P. (2015). "Analytical Network Process (ANP) to Recommend an Ice Cream to a Diabetic Patient". International Journal of Computer Applications, 121(12), 49-52. doi: 10.5120 /21596-4692

[21] Gaikwad, S. M., Joshi, R. R., \& Kulkarni, A. J. (2016). "Cohort Intelligence and Genetic Algorithm Along with Modified Analytical Hierarchy Process to Recommend an Ice Cream to Diabetic Patient". Proceedings of the International Conference on Data Engineering and Communication Technology Advances in Intelligent Systems and Computing, 279-288. doi: 10.1007/978-981-10-1675-2_29

[22] Gaikwad, S. M., R., R., \& Mulay, P. (2015). "Cluster Mapping with the help of New Extended MCF Algorithm and MCF Algorithm to Recommend an Ice Cream to the Diabetic Patient". International Journal of Computer Applications, 127(6), 25-27. doi: 10.5120/ijca2015906399

[23] S. M. Gaikwad, R. R. Joshi, Mulay, P., "Attribute visualization and cluster mapping with the help of new proposed algorithm and modified cluster formation algorithm to recommend an ice cream to the diabetic patient based on sugar contain in it," vol. 10, pp. 1-6, 2015.

[24] V. M. Jossy P George, "Mobile in learning: Enhancement of information and communication technologies," International Journal of Engineering \& Technology, vol. 7, no. 2, pp. 98-101, Mar. 2018. 


\section{BIOGRAPHIES OF AUTHORS}
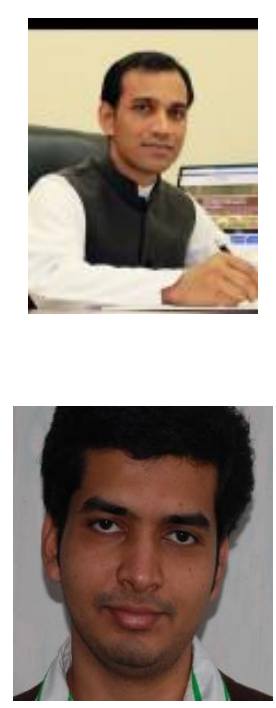

Dr. (Fr) Jossy P. George has dual Master degree in Computer Science and Human Resources from USA and has done his FDPM from IIM, Ahmedabad. He has been awarded a Doctorate in Computer Science by Christ University, Bengaluru on Image Processing titled "Development of Efficient Biometric Recognition Algorithms Based on Fingerprint And Face". He is a member of International Association of Computer Science and Information Technology (IACSIT) and Computer Society of India (CSI). His research activities focus on algorithms for improved accuracy in biometrics. He has been working with Christ University (previously Christ College), Bengaluru, India and other associated institutions since 2002 in various capacities and is currently serving as Director and Dean of CHRIST (Deemed to be University), Lavasa, Pune

$\mathrm{He}$ is the researcher at Christ Deemed to be University Lavasa, Pune, India. Has Master degree in Automotive System From Holland also M.Tech degree in Computer Science from Symboisis Institute. He is internationally renowed Engineer, Scientist. Serving as an Editor borad member for couple of Journal from Medical Science as well as from Enginerring. His research interest are Optimization, AHP, ANP, and System Dynamics, Vehicle Dynamics, Data Mining, Clustring. Artifitail Intelligance, Genetic Algorithm. Also, Publsihed sevral research article in daomian of Soft computing. 